Journal of

Epilepsy and

Clinical

Neurophysiology

J Epilepsy Clin Neurophysiol 2005; 11(3):131-136

\title{
A Disfunção do Lobo Frontal em Crianças e Adolescentes com Epilepsia de Lobo Temporal e sua Possível Correlação com a Ocorrência de Transtornos Psiquiátricos
}

\author{
Patrícia Rzezak*, Daniel Fuentes*, Catarina A. Guimarães**, Marilisa Guerreiro**, \\ Kette D. R. Valente*** \\ Laboratório de Neurofisiologia, Instituto e Departamento de Psiquiatria - Hospital das Clínicas/USP
}

\begin{abstract}
RESUMO
Introdução: Há evidências de que pessoas com alguns tipos de epilepsia podem apresentar prejuízos cognitivos, sendo que para alguns pacientes, tais déficits cognitivos podem ser mais debilitantes do que suas crises epilépticas. A disfunção do lobo frontal é relatada em adultos com epilepsia do lobo temporal, caracterizada pela disfunção executiva. Objetivo: Rever a literatura sobre a ocorrência de disfunção de lobo frontal em crianças e adolescentes com epilepsia do lobo temporal. Métodos: Revisão sistemática da literatura compulsada no PUBMED. Resultados: A disfunção do lobo frontal tem sido pouco estudada em crianças com epilepsia, em especial com epilepsia do lobo temporal. No único estudo sobre este assunto, demonstrouse que há um déficit executivo, sendo que as crianças com esclerose mesial têm maiores déficits de execução/ planejamento do que aquelas com lesões neocorticais temporais ou frontais. A presença de uma disfunção do lobo frontal nos pacientes com epilepsia parcial e generalizada pode corroborar as evidências de que epilepsia e transtorno psiquiátrico são epifenômenos e não fenômenos com relação causa-efeito. Conclusões: Há uma escassez de artigos sobre a disfunção do lobo frontal em crianças com epilepsia do lobo temporal, sendo de interesse saber se esta ocorre e se pacientes com etiologias distintas apresentam diferentes graus de comprometimento destas funções.
\end{abstract}

Unitermos: epilepsia, crianças, lobo frontal, lobo temporal, transtornos psiquiátricos.

\begin{abstract}
Frontal lobe disfunction in children and adolescents with temporal lobe epilepsy and possible correlation with psychiatric disorders

Introduction: There is evidence that individuals with certain types of epilepsy may present cognitive disorders, and that these disorders can be more debilitating than seizures proper. Frontal lobe disorders are reported in adults with temporal lobe epilepsy, carachterized by executive disfunction. Rationale: Literary revision of work concerning the occurence of frontal lobe dysfunction in children and adolescents with temporal lobe epilepsy. Methods: Systematic revision of published literature in PUBMED. Results: Frontal lobe dysfunction has been poorly studied in children with epilepsy, especially in temporal lobe epilepsy. In the only study on the subject, executive deficit was demonstrated. Moreover, children with mesial sclerosis had more deficit in execution/planning than those with frontal or temporal neocortical lesions. Presence of frontal lobe dysfunction, in patients with partial and generalized epilepsy, may corroborate evidence that epilepsy and psychiatric disorders are epiphenomena and not cause-effect related phenomena. Conclusion: There are few articles on frontal lobe dysfunction in children with temporal lobe epilepsy. It would be interesting to find out if, and how much this occurs, and if patients with distinct etiologies present different levels of functional disability.
\end{abstract}

Key words: epilepsy, children, frontal lobe, temporal lobe, psychiatric disorders.

\footnotetext{
* Unidade de Neuropsicologia da Divisão de Neurocirurgia Funcional do Instituto de Psiquiatria do HC-FMUSP.

** Departamento de Neurologia da UNICAMP.

*** Laboratório de Neurofisiologia - Projeto para Estudo dos Transtornos Psiquiátricos em Crianças e Adolescentes com Epilepsia - Instituto e Departamento de Psiquiatria do Hospital das Clínicas da Universidade de São Paulo.

Received Feb 14, 2005; accepted Apr 25, 2005.
} 


\section{INTRODUÇÃO}

Há evidências de que pessoas com alguns tipos de epilepsia podem apresentar prejuízos cognitivos, sendo que para alguns pacientes, tais déficits cognitivos podem ser mais debilitantes do que suas crises epilépticas ${ }^{[1]}$. Tendo isto em vista, nota-se a importância da realização de avaliações neuropsicológicas nesta população, com o objetivo de diagnosticar tais prejuízos cognitivos e acessar o impacto destas disfunções cognitivas no dia-a-dia.

As funções cognitivas, ou seja, a capacidade de processar adequadamente as informações e de programar o comportamento adaptativo depende do processamento cortical e envolve inúmeras funções, entre elas, a habilidade de resolver problemas, memorizar informações, focar a atenção, além de estar relacionada com a capacidade de lidar, de forma criativa, com situações complexas, transcendendo a situação imediata e antecipando a ação fu$\operatorname{tura}^{(1)}$.

\section{FUNÇÕES COGNITIVAS NA EPILEPSIA DE LOBO TEMPORAL NOS ADULTOS}

\section{- Déficits mnésticos}

Na epilepsia de lobo temporal (ELT), é reconhecida a presença de déficits cognitivos específicos. É comum que estes pacientes apresentem disfunções de memória, principalmente quando há o comprometimento de ambos os lobos temporais, ou da linguagem quando a zona epileptogênica está localizada no lobo temporal dominante para a linguagem ${ }^{(2)}$.

Existem evidências de que os pacientes com ELT unilateral apresentam déficits neuropsicológicos lateralizados. Foi postulado que tais déficits podem ser causados pela perda de células associada com a lesão ou pela interferência das descargas epileptiformes interictais na cognição ${ }^{(3)}$.

Há estudos ${ }^{(3-5)}$ que tentam diferenciar a lateralização da memória verbal e não-verbal. Entretanto, parece haver uma dificuldade na interpretação das diferenças interhemisféricas, o que estaria relacionado com o fato da lateralidade das funções de memória dependerem da lateralidade da linguagem ${ }^{(4)}$.

Kim et al. ${ }^{(4)}$ e Hermann et al. ${ }^{(6)}$ relatam que indivíduos com dominância hemisférica para a linguagem à esquerda costumam apresentar a memória para informações verbais no hemisfério esquerdo e a memória para material visuoespacial no hemisfério direito. Contudo, estes autores discordam quanto a lateralização da memória quando a dominância hemisférica para linguagem não é no hemisfério esquerdo. Kim et al. ${ }^{(4)}$ descreveram que este padrão de lateralização da memória não foi encontrado naqueles indivíduos que não possuem dominância hemisférica para linguagem à esquerda, enquanto que Hermann et al. ${ }^{(6)}$ relataram o comprometimento da memória visuoespacial quando o foco epileptogênico é no hemisfério direito, favorecendo a hipótese da lateralização da memória quando a linguagem não está no hemisfério esquerdo.

A esclerose mesial temporal é freqüentemente associada a prejuízos de memória relacionado com o fato da zona epileptogênica, bem como a lesão, localizar-se em uma região importante na consolidação de novas memórias $^{(4,7)}$. As estruturas temporais mesiais estão relacionadas com o processamento da memória declarativa de longo prazo no período de desenvolvimento da criança. Isto significa que uma lesão precoce nestas regiões não pode ser compensada pela ativação de regiões alternativas e que o grau de prejuízo mnéstico é relacionado à idade em que a criança adquiriu a lesão ${ }^{(7)}$.

Vê-se, portanto, que lesões nas regiões mesiais do lobo temporal são suficientes para originar os déficits de memória. No entanto, existem indícios de que não apenas a formação hipocampal, mas também, as regiões neocorticais adjacentes estão envolvidas no processamento da memória. Cabe, então, questionar-se se estas regiões neocorticais também são importantes para as funções de memória ${ }^{(8)}$.

Em um estudo realizado por Cheung e Chan ${ }^{(8)}$ foi possível encontrar evidências da participação do neocórtex na memória, quando se estudou um grupo de pacientes com déficits mnésticos após lesão bilateral no córtex temporal lateral, com preservação hipocampal. Interessantemente, os déficits desses pacientes com lesões neocorticais temporais bilaterais eram comparáveis aos daqueles com lesões hipocampais.

\section{- Disfunção de lobo frontal}

A disfunção do lobo frontal (DLF) é relatada em adultos com ELT, caracterizada pela disfunção executiva. Como função executiva, entende-se as capacidades que permitem que o indivíduo realize, com sucesso, ações independentes, propositadas e auto-determinadas. Elas podem ser conceitualizadas como contendo quatro fatores: a volição, o planejamento, a ação propositada e a performance efi$\mathrm{caz}^{(9)}$. Assim, envolvem o planejamento, a utilização de experiências passadas, a iniciação da ação, a manutenção e monitorização da ação e a capacidade de mudar e inibir respostas comportamentais ${ }^{(10)}$. Desta forma, enquanto as funções executivas estiverem intactas, o indivíduo poderá (mesmo com perdas cognitivas consideráveis) continuar a ser independente. Entretanto, se tais capacidades estiverem prejudicadas, o indivíduo terá dificuldades em iniciar uma ação, diminuição da motivação, defeitos de planejamento e manutenção da seqüência de atividades necessária à realização da meta ${ }^{(9)}$. Tais atividades parecem estar relacionadas com o circuito dorsolateral ${ }^{(10)}$.

Portanto, a DLF inclui prejuízos na habilidade em resolver problemas, na capacidade de abstração e na existência da tendência à perseveração ${ }^{(11,12)}$. No momento, 
existem duas hipóteses para explicar esta disfunção frontal em pacientes com ELT, que serão detalhadas a seguir.

Segundo Corcoran e Upton ${ }^{(13)}$, a presença de esclerose hipocampal em hemisfério direito (EH) estaria associada a uma pior performance em teste avaliador de flexibilidade mental (Wisconsin Card Sorting Test - WCST), o que foi demonstrado pelo pior desempenho destes pacientes do que aqueles com epilepsia neocortical ou, até mesmo, pacientes com epilepsia frontal. Portanto, esta disfunção de lobo frontal seria explicada pela lesão hipocampal à direita, e estaria relacionada com a memória operacional (working memory). Esta dificuldade era esperada considerando o comprometimento não verbal working memory no WCST. Em concordância ao achado de Corcoran e Upton $^{(13)}$, o estudo de Hermann, Wyler e Ritchie ${ }^{(14)}$ demonstrou um pior desempenho dos pacientes com foco no lobo temporal direito.

Contudo, não existe ainda um consenso quanto ao desempenho no WCST e a lateralização da lesão. Horner et al. ${ }^{(15)}$ não encontraram diferenças significativas entre pacientes com foco no lobo temporal direito e esquerdo. Além disso, Hermann e Seidenberg ${ }^{(11)}$, não encontraram associação entre a presença de esclerose mesial e a performance no WCST, postulando que a atividade epileptogênica anormal do LT, independentemente da etiologia, afetaria regiões extratemporais que mediam as habilidades executivas (frontais), o que justificaria a melhora nestas funções após a lobectomia temporal anterior.

\section{FUNÇÕES COGNITIVAS NA EPILEPSIA DO LOBO TEMPORAL NA INFÂNCIA}

A avaliação neuropsicológica ganha uma importância ainda maior quando se trata da faixa etária pediátrica. Isso porque, nesta fase, ainda é possível a mudança do prognóstico, através da reabilitação neuropsicológica. Além disso, auxilia na escolha de um tratamento efetivo, prevenindo assim disfunções cognitivas secundárias à epilepsia e uma vida adulta com possibilidades ocupacionais restri$\operatorname{tas}^{(1)}$.

\section{- Alterações mnésticas}

Quanto à avaliação neuropsicológica em crianças com ELT, a relação entre a performance em testes neuropsicológicos e a lateralização do foco epileptogênico ainda é controversa. Adams et al. ${ }^{(16)}$ observaram prejuízos iguais quanto à memória verbal e visual em crianças com foco à direita e à esquerda. Por outro lado, Jambaque et al. ${ }^{(17)}$ demonstraram déficits de memória verbal em crianças com ELT à esquerda e de memória visual nas com ELT à direita, equivalente ao encontrado nos adultos com esta síndrome.

No estudo de Lendt et al. ${ }^{(18)}$, com 20 crianças e préadolescentes com ELT (idade média de 13,8 anos), as fun- ções mnésticas estavam intactas. Este dado é contrário ao que é freqüentemente observado em adultos com ELT. Os autores creditam isso ao menor tempo de duração da epilepsia em crianças, o que reduziria a ocorrência de déficits cognitivos.

No estudo de Szabó et al. ${ }^{(19)}$, com 14 pré-adolescentes com ELT (idade média de 9,4 anos), foram realizadas avaliações neuropsicológicas pré e pós-operatórias e constatou-se a estabilidade do QI e a diminuição da memória verbal após ressecção do lobo temporal na infância. A relativa estabilidade das medidas de QI em pré-adolescentes indica que a habilidade intelectual geral não é intensamente afetada pela ressecção do lobo temporal.

Segundo Szabó et al. ${ }^{(19)}$, poucos estudos avaliaram os efeitos da ELT e da lobectomia temporal na cognição de crianças com epilepsia. Na revisão realizada por estes autores, a maior parte das séries abordando este tópico tem resultados semelhantes aos dos estudos com adultos em conseqüência da população estudada, predominantemente composta por adolescentes, limitando as conclusões sobre as disfunções mnésticas em crianças pós-cirurgia do lobo temporal.

\section{- Disfunção de lobo frontal}

No único estudo sobre DLF em crianças com ELT, Igarashi et al. ${ }^{(20)}$ relataram que as crianças com esclerose mesial têm maiores déficits de execução/planejamento do que aquelas com lesões neocorticais temporais ou frontais. No entanto, este estudo incluiu pacientes com idades de até 20 anos (que podem ser considerados adultos), além de ser composto por uma casuística heterogênea com subgrupos de poucos pacientes com esclerose mesial e lesão neocortical. O que também pode ser observado é que os pacientes com ELT foram comparados com aqueles com lesões em lobos frontais em que não foi especificado o tipo de lesão ou a sua localização (córtex motor, área motora suplementar ou córtex pré-frontal). Por fim, a bateria de testes neuropsicológicos foi pouco abrangente, limitando-se apenas a um teste, creditando uma inadequada especificidade teste-função.

Portanto a ocorrência de DLF em crianças com epilepsia precisa ser esclarecida. Resultados preliminares do estudo colaborativo em andamento na Universidade de Campinas e na Universidade de São Paulo, vêm demonstrando que crianças com ELT tanto mesial quanto neocortical apresentam falhas executivas, com pior performance nas tarefas de controle inibitório e de flexibilidade mental nas crianças com lesões neocorticais do que naquelas com lesões mesiais. Assim, estamos percebendo que os dois grupos de crianças com ELT (mesial e neocortical) apresentam disfunção executiva, caracterizada por falhas no controle dos impulsos e maiores dificuldades de abstração e formação de conceitos, e que isto 
ocorre mais acentuadamente nas crianças com envolvimento das regiões neocorticais. Estes resultados foram obtidos utilizando-se uma ampla bateria de testes neuropsicológicos que incluiu o Matching Familiar Figures Test, o Wisconsin Card Sorting Test e o Trail Making infantil.

\section{A POSSÍVEL INTER-RELAÇÃO ENTRE OS TRANSTORNOS PSIQUIÁTRICOS E A DISFUNÇÃO DO LOBO FRONTAL}

Nota-se um aumento do interesse sobre a comorbidade entre a epilepsia e os transtornos psiquiátricos ${ }^{(21)}$. Sintomas de depressão, ansiedade, medo, labilidade/instabilidade emocional, mania, confusão, alucinações e ilusões, que ocorrem isoladamente ou em combinação com fenômenos ictais. Como uma das comorbidades mais freqüentemente observada e estudada entre os adultos, a depressão atinge uma prevalência de $8 \%$ a $48 \%$ em diferentes estudos, o que caracteriza uma maior freqüência em pacientes com epilepsia do que na população em geral ${ }^{(21)}$.

A presença de transtornos psiquiátricos em crianças com epilepsia, no entanto, continua sendo pouco diagnosticada e conseqüentemente pouco tratada. No estudo de Ott et al. ${ }^{(22)}$, aproximadamente $60 \%$ dos pacientes com epilepsia apresentavam um transtorno psiquiátrico e mais de $60 \%$ destes não tinham nenhum diagnóstico psiquiátrico prévio. Em um estudo recente ${ }^{(23)}$ foi possível observar que a depressão e o déficit de atenção e hiperatividade são distúrbios comuns, sendo a primeira mais freqüente entre os adolescentes e a segunda entre as crianças. Portanto, parece haver uma expressão idade-dependente de transtornos psiquiátricos em crianças com epilepsia. Outros fatores, como o tipo de epilepsia e localização do foco são importantes na determinação da freqüência e do tipo de transtorno psiquiátrico. Dentre as epilepsias sintomáticas, a maior causa de epilepsia refratária no adulto é a ELT e a presença de transtornos psiquiátricos é freqüente, sendo que a incidência de depressão nestes é maior do que naqueles com epilepsia generalizada ou com foco extratemporal ${ }^{(24)}$. Não temos conhecimento se este dado encontra-se replicado na infância.

Segundo Bortz et al. ${ }^{(21)}$ os transtornos psiquiátricos nos pacientes com epilepsia poderiam ser ocasionados por três diferentes fatores: (i) presença de uma alteração estrutural e funcional no SNC que acabaria por mediar a co-existência de duas doenças mentais orgânicas; (ii) como conseqüência de fatores psicológicos relacionados com as limitações impostas pelas crises epilépticas ou pelo estigma social gerado pela doença; e (iii) como efeito adverso das drogas antiepilépticas (DAE).

O lobo frontal tem sido objeto de estudo nos pacientes com transtornos psiquiátricos. Considerando-se a presença de um mecanismo comum que subsidie a co-existência da epilepsia e do transtorno psiquiátrico, a presen- ça de uma DLF nos pacientes com epilepsia parcial e generalizada pode corroborar as evidências de que epilepsia e transtorno psiquiátrico são epifenômenos e não fenômenos com relação causa-efeito ${ }^{(24)}$. Estudos de neuroimagem e de neurotransmissores corroboram esta hipótese.

\section{- Estudos de neuroimagem}

Estudos com métodos de neuroimagem convencionais vêm fornecendo evidências de que há uma relação com a região do foco, sendo que a depressão foi identificada mais freqüentemente quando a epilepsia tinha sua origem no lobo temporal ou frontal, isto é, quando estruturas do circuito límbico estava envolvido.

Em relação à lateralidade, estudos com PET (photon emision tomography) e SPECT (single photon emision computadorized tomography) têm demonstrado metabolismo diminuído e menor perfusão cerebral, respectivamente, no hemisfério esquerdo nos pacientes com epilepsia parcial e depressão interictal ${ }^{(25)}$.

A DLF vem sendo demonstrada através de estudos de neuroimagem funcional e avaliação neuropsicológica em pacientes com epilepsia do lobo temporal e depressão. Estudos com PET têm demonstrado uma diminuição do metabolismo do lobo frontal em pacientes com ELT e os estudos neuropsicológicos mostram um declínio das funções executivas, através do WCST, em pacientes com ELT e depressão ${ }^{(26)}$.

Existem, entretanto, poucos estudos com métodos de neuroimagem mais requintados tentando estabelecer uma correlação entre as alterações observadas na neuroimagem e a presença de transtornos mentais em adultos e crianças com epilepsia.

Gilliam et al. ${ }^{(27)}$ utilizam a espectroscopia para estudar a associação entre a intensidade das anormalidades observadas à espectroscopia (1H MRS) na esclerose mesial temporal e a presença de transtornos do humor e qualidade de vida. Para tal avaliaram 33 adultos - idade média de 35 anos com idade de início da epilepsia de 15 anos - com epilepsia refratária e ELT. A extensão da relação $\mathrm{N}$-acetilaspartato/creatina (NAA/Cr) e $\mathrm{N}$-acetilaspartato/colina (NAA/Cho) nos lobos temporais (incluindo estruturas mesiais e o neocórtex) esteve diretamente associada à presença de depressão. Outras variáveis relacionadas à epilepsia e funcionamento psicossocial, como a freqüência de crises, quantidade de medicações, emprego, capacidade de dirigir veículos, não se associaram à presença de depressão. A presença de alterações neocorticais em adultos com ELT, detectadas através da espectroscopia, relaciona-se com a ocorrência de depressão. Uma abordagem crítica deste trabalho demonstra que o estudo limitado às estruturas temporais pela ressonância magnética representou um fator limitante para a melhor análise do comprometimento das estruturas extratemporais ${ }^{(27)}$. 


\section{- Neurotrasmissores}

A diminuição da atividade e da função serotoninérgica (5HT), noradrenérgica (NE), dopaminérgica (DA) e gabaérgica (GABA), sintetizados no lobo frontal, vem sendo identificada como pivô dos mecanismos patogênicos da depressão e tem sido a base para o tratamento farmacológico antidepressivo ${ }^{(28)}$.

Uma diminuição da atividade destes neurotransmissores tem sido demonstrada como um facilitador no processo de abrasamento do foco epileptogênico, na exacerbação da gravidade das crises, e na intensificação da predisposição a crises epilépticas em alguns modelos animais $^{(29)}$.

O papel dos neurotransmissores em modelos animais de epilepsia pode ser observado através dos estudos com duas subespécies de ratos geneticamente predispostos à epilepsia (GEPRs), GEPR-3 e GEPR-9, servem como modelo ideal de epilepsia parcial. Crises induzidas pelo som no GEPR-9 mimetizam as crises secundariamente generalizadas em humanos ${ }^{(30-31)}$. Ambos os modelos (GEPR-3 e GEPR-9) apresentam déficits de transmissão pré e póssinápticas serotoninérgicas, que têm sido correlacionados com a predisposição a crises epilépticas nestes ratos. Os ratos GEPR-9 apresentam um déficit de transmissão NE mais pronunciado, e, portanto exibem crises mais graves do que os ratos GEPR-3(31).

Aumentos da transmissão da NE e/ou da $5 \mathrm{HT}$ podem prevenir a ocorrência de crises; por outro lado, a sua redução tem um efeito oposto, aumentando as crises epilépticas $^{(32)}$.

O uso de inibidores da recaptação da serotonina (ISRS) e dos inibidores da mono-amino-oxidase (IMAOs) exercem um efeito anticonvulsivante nestes camundongos geneticamente predispostos à epilepsia ${ }^{(31)}$ e nos macacos babuínos ${ }^{(31-32)}$, assim como em outros animais não-geneticamente predispostos: em gatos ${ }^{(33)}$, $\operatorname{coelhos}^{(34)}$ e macacos Rhesus $^{(35)}$.

O impacto das drogas antidepressivas em pessoas com epilepsia não é tão marcante como o observado em modelos animais. Entretanto, há relatos da supressão de crises de ausência e crises mioclônicas com o uso da imipramina, um antidepressivo tricíclico com efeito inibitório na recaptação da NE e 5HT(37-39).

Portanto, mudanças paralelas que podem ser identificadas na serotonina $(5 \mathrm{HT})$, norepinefrina $(\mathrm{NE})$, dopamina (DA), e ácido g-aminobutírico (GABA) podem ser operantes na fisiopatologia da depressão e da epilepsia, o que indica que a epilepsia e a depressão podem ter mecanismos etiopatogênicos comuns mediados por uma diminuição da atividade de alguns neurotransmissores ${ }^{(24)}$, sendo que a ocorrência de um pode facilitar o desenvolvimento do outro e vice-versa.
Esperamos que o estudo que ora temos em andamento possa vir a lançar luz a esta e a outras questões importantes nas relações entre epilepsia e disfunção cognitiva em crianças e adolescentes.

\section{CONCLUSÃO}

A avaliação neuropsicológica dos pacientes com epilepsia do lobo temporal tem enfatizado o estudo das funções mnésticas. Entretanto, tornam-se claras que as disfunções cognitivas não estão restritas à área lesada, envolvendo outras regiões como o lobo frontal. A disfunção do lobo frontal pode ser decorrente do possível comprometimento de vias hipocampais-frontais ou através da propagação de paroxismos epileptiformes que prejudicam o desempenho de testes que avaliam funções frontais em pacientes com epilepsia do lobo temporal, o que permanece a ser determinado. As implicações deste acometimento estão relacionadas à menor capacidade de execução e planejamento observada nestes pacientes, assim como pode estar relacionada a maior ocorrência de outras comorbidades, como os transtornos psiquiátricos.

\section{REFERÊNCIAS}

1. ILAE - Diagnosis. Clinical Neuropsychology: Cognitive Function in Epilepsy. Epilepsia 2003; 44(6):29-30.

2. Blume WT. Diagnosis and management of epilepsy. CMAJ 2003; 168(4):441-8.

3. Akanuma $\mathrm{N}$ et al. Lateralising Value of Neuropsychological Protocols for Presurgical Assessment of Temporal Lobe Epilepsy. Epilepsia 2003; 44(3):408-18.

4. Kim H et al. Material- Specific Memory in Temporal Lobe Epilepsy: Effects of seizure Laterality and Language Dominance. Neuropsychology 2003; 17 (1):59-68.

5. Kim H et al. Differential Effects of left versus right mesial temporal lobe epilepsy on Wechsler Intelligence Factors. Neuropsychology 2003; $17(4):$ 556-65.

6. Hermann BP, Seidenberg M, Schoenfeld, J, Davis K. Neuropsychological characteristics of the syndrome of Mesial Temporal Lobe Epilepsy. Arch Neurol 1997; 54(4):369-76.

7. Riva D et al. Memory disturbance in early hippocampal pathology. In: Avanzini $G$ et al. Limbic seizures in Children. Milão: John Libbey; 2001. p.167-74.

8. Cheung M e Chang AS. Memory impairment in humans after bilateral damage to lateral temporal neocortex. Neuroreport 2003; $14(3): 371-74$.

9. Lezak MD. Neuropsychological Assessment. 3a ed. Nova Iorque: Oxford University Press; 1995. p.42; 621-5.

10. Doval O, Gaviria M, Kanner AM. Frontal lobe dysfunction in epilepsy. In: Ettinger AB, Kanner AM, editors. Psychiatric Issues in epilepsy - a practical guide to diagnosis and treatment. Filadélfia: Lippincott Williams \& Wilkins; 2001. p. 261-71.

11. Hermann BP, Seidenberg M. Executive system dysfunction in temporal lobe epilepsy: effects of nociferous cortex versus hippocampal pathology. J Clin Exp Neuropsychol 1995; 7:809-19.

12. Trenery M, Jack Jr CR. Wisconsin Card Sorting Test performance before and after temporal loberctomy. J Epilepsy 1994; 7:313-7.

13. Corcoran R, Upton D. A role for the hippocampus in card sorting? Cortex 1993; 29:293-304.

14. Hermann BP, Wyler AR, Ritchie ET. Wisconsin Card Sorting Test performance in patients with complex partial seizures of temporal lobe origin. J Clin Neuropsychol 1988; 10:467-76. 
15. Horner MD, Flashman LA, Freides D. Focal epilepsy and the Wisconsin Card Sorting Test. J Clin Exp Neuropsychol 1989; 11:74.

16. Adams CBT et al. Temporal lobectomy in 44 children: outcome and neuropsychological follow-up. J. Epilepsy 1990; 3(1): 157-68.

17. Jambaque I et al. Verbal and visual memory impairment in children with epilepsy. Neuropsychologia 1993; 314:1321-37.

18. Lendt M, Helmstaedter C, Elger CE. Pre-and postoperative neuropsychological profiles in children and adolescents with temporal lobe epilepsy. Epilepsia 1999; 40(11):1543.

19. Szabó CA. Neuropsychological effect of temporal lobe resection in preadolescent children with epilepsy. Epilepsia 1998; 39(8):814-19.

20. Igarashi K, Oguni H, Osawa M, Yutaka A et al. Wisconsin Card Sorting Test in children with Temporal Lobe Epilepsy. Brain \& Devel 2002; 24(3):174-8.

21. Bortz JJ. Neuropsychiatry and memory issues in epilepsy. Epilepsy 2003; 78(6):781-7.

22. Ott D et al. Behavioral Disorders in Pediatric Epilepsy: Unmet Psychiatric Need. Epilepsia 2003; 44(4):591-7.

23. Thome-Souza S, Kuczynski E, Assumpcao Jr F, Rzezak P, Fuentes D, Fiore L, Valente KD. Which factors may play a pivotal role on determining the type of psychiatric disorder in children and adolescents with epilepsy? Epilepsy Behav. 2004 Dec; 5(6):988-94.

24. Kanner AM, Balabanov A. Depression and epilepsy: how closely related are they? Neurology 2002 Apr 23; 58(8 Suppl 5):S27-39.

25. Gadian DG, Isaacs EB, Cross JH, Connelly A, Jackson GD, King MD, Neville BGR, Vargha-Khadem F. Lateralization of brain fuction in childhood revealed by magnetic resonance spectroscopy. Neurology 1996; 46:974-77.

26. Hermann B, Seidenberg M, Bell B, Rutecki P, Sheth R, Ruggles K, Wendt G, O'Leary D, Magnotta V. The neurodevelopmental impact of childhood onset temporal lobe epilepsy on brain structure and fuction. Epilepsia 2002; 43(9):1062-71.

27. Gilliam F, Maton B, Martin RC, et al. Extent of $1 \mathrm{H}$ spectroscopy abnormalities independently predicts mood status and quality of life in temporal lobe epilepsy. Epilepsia 2000; 41(Suppl 7):54.
28. Schildkraut JJ. The catecolamine hypothesis of affective disorders: a review of supporting evidence. Am J Psychiatry 1965; 122:509-522.

29. Jobe PC, Dailey JW, Wernick JF. A noradrenergic and serotonergic hypothesis of the linkage between epilepsy and affective disorders. Crit Rev Neurobiol 1999; 13:317-56.

30. Jobe PC, Mishra PK, Adams-Curtis LE, et al. The genetically epilepsy-prone rat (GEPR). Ital J Neurol Sci 1995; 16:91-9.

31. Lehmann A. Audiogenic seizures data in mice supporting new theories of biogenic amines mechanisms in the central nervous system. Life Sci 1967; 6:1423-31.

32. Meldrum BS, Anlezark GM, Adam HK, Greenwod DT. Anticonvulsivant and proconvulsant properties of viloxazine hydrochloride: pharmacological and pharmacokinetic studies in rodents and epileptic baboon. Psychopharmacology (Berl) 1982; 76:212-7.

33. Polc P, Scheeberg J, Haefely W. Effects of several centrally active drugs on the sleep-wakefulness cycle of cats. Neuropharmacology 1979; 18:259-67.

34. Piette Y, Delaunois AL, De Shaepdryver AF, Heymans C. Imipramine and electroshock threshold. Arch Int Pharmacodyn Ther 1963; 144:293.

35. Yanagita T, Wakasa Y, Kiyohara H. Drug dependence potential of viloxazine hydrochloride tested in rhesus monkeys. Pharmacol Biochem Behav 1980; 12:155-61.

37. Fromm GH, Rosen JA, Amores CY. Clinical and experimental investigation of the effect of imipramine on epilepsy. Epilepsia 1971; $12: 282$.

38. Fromm GH, Wessel HB, Glass JD, Alvin JD, Van Horn G. Imipramine in absence and myoclonic-astatic seizures. Neurology 1978; 28:953-7.

39. Fromm GH, Amores CY, Thies W. Imipramine in epilepsy. Arch Neurol 1972; 27:198-204.

Endereço para correspondência:

Kette D. R. Valente

Rua Jesuíno Arruda, 901

CEP 04532-082, São Paulo, SP, Brasil 